\title{
The Blind Passenger and the Assignment Problem
}

\author{
JOHAN WÄSTLUND \\ Department of Mathematical Sciences, Chalmers University of Technology, S-412 96 Gothenburg, Sweden \\ (e-mail: wastlund@chalmers.se)
}

Received 27 November 2008; revised 19 November 2010; first published online 14 February 2011

\begin{abstract}
We introduce a discrete random process which we call the passenger model, and show that it is connected to a certain random model of the assignment problem and in particular to the so-called Buck-Chan-Robbins urn process. We propose a conjecture on the distribution of the location of the minimum cost assignment in a cost matrix with zeros at specified positions and remaining entries of exponential distribution. The conjecture is consistent with earlier results on the participation probability of an individual matrix entry. We also use the passenger model to verify a conjecture by V. Dotsenko on the assignment problem.
\end{abstract}

\section{The blind passenger problem}

During work with the Swedish mathematical 'Olympiad' in 2004, I was challenged with an amusing problem by Victor Ufnarovski and Dag Jonsson, two other members of the problem committee, who had heard it through the grapevine. In the problem, $n$ passengers are boarding an aircraft. Each passenger has a ticket to one of the $n$ numbered seats, but one passenger is blind and therefore cannot read the ticket. The blind passenger boards the aircraft first, and chooses a seat at random. Then the remaining passengers enter one by one. A passenger whose seat is free will choose that seat. A passenger whose seat is already occupied by another person will choose a free seat at random. All random choices are uniform and independent. The question is: What is the probability that the last passenger gets his or her own seat? The neat answer is $1 / 2$, and the reason is that the tickets of the blind passenger and the last passenger do not affect the process. When the last passenger enters, all seats other than those two must be occupied, and therefore the free seat is either the one to which the last passenger has a ticket, or to which the blind passenger has a ticket, with equal probability.

While thinking about this problem and its various generalizations, I recognized some numbers that occurred in my calculations from a seemingly different context. This led to a slightly different model of the passengers entering the aircraft.

Before describing this model, we make a brief outline of the paper. The next section describes a passenger model, which can be coupled to the Buck-Chan-Robbins urn 
process $[16,20]$ in such a way that passenger-seat pairs without tickets correspond to so-called outer corners in the urn process. The urn process was invented because of its connection to random assignment problems, and in particular it is known [16] that the participation probability of a matrix entry in a random assignment problem with prescribed zero entries is equal to the probability of a corresponding outer corner in the urn process. We conjecture that the set of matrix positions in the solution to the assignment problem is equal in distribution to the set of outer corners in the urn process and thereby to the set of unticketed passenger-seat pairs.

We apply the passenger model in order to prove several properties of the random assignment problem, among them the Parisi formula (3.1), although this is not strictly a new proof since it relies on the previously established correspondence between the assignment problem and the urn process. We also justify a claim by Dotsenko [5].

\section{The passenger model}

We consider $n$ passengers boarding an aircraft with $n$ seats. Some of the passengers have a ticket to one of the seats, while others do not have a ticket. We allow several passengers to have tickets to the same seat. The passengers are seated by a random process in two stages. First they are ordered by a uniformly chosen permutation. Then they enter the aircraft one by one. A passenger who has a ticket to a seat will choose that seat if it is free. A passenger whose seat is already occupied, or who does not have a ticket, will choose uniformly between the free seats. All random choices are made independently of each other.

If we number the passengers from 1 to $n$ and likewise the seats from 1 to $n$, the initial data for the process can be represented by a subset $Z \subseteq[n] \times[n]$, where $(i, j) \in Z$ means that passenger $i$ has a ticket to seat $j$. We let $P(i, j, Z)$ denote the probability that passenger $i$ ends up in seat $j$.

Example 2.1. Suppose $n=4$, and $Z=\{(1,2),(2,1),(3,1)\}$, in other words, passenger 1 has a ticket to seat 2 , passengers 2 and 3 both have tickets to seat 1 , and passenger 4 does not have a ticket. The situation can be visualized by the following matrix, where rows correspond to passengers, columns to seats, and bullets represent tickets:

$$
\left(\begin{array}{c}
-\bullet-- \\
\bullet--- \\
\bullet--- \\
----
\end{array}\right) \text {. }
$$

There are a number of cases to consider since we are averaging both over the order in which the passengers enter, and over the way they choose their seats. A brute force calculation shows that the probabilities $P(i, j, Z)$ are given by the matrix

$$
\left(\begin{array}{cccc}
\frac{1}{144} & \frac{35}{48} & \frac{19}{144} & \frac{19}{144} \\
\frac{65}{144} & \frac{1}{16} & \frac{35}{144} & \frac{35}{144} \\
\frac{65}{144} & \frac{1}{16} & \frac{35}{144} & \frac{35}{144} \\
\frac{13}{144} & \frac{7}{48} & \frac{55}{144} & \frac{55}{144}
\end{array}\right) .
$$


I recognized the numbers occurring in tables like (2.2) from a different context, random assignment problems.

\section{Random assignment problems}

Consider an $n$ by $n$ matrix of non-negative real numbers. An assignment is a set of matrix positions, one in each row, of which no two are in the same column. The cost of an assignment is the sum of the corresponding matrix entries. The assignment problem is the optimization problem asking for the minimum cost assignment. If the matrix entries are random variables, then so is the cost of the minimum assignment. A problem that has received considerable attention (see [1] for further references) is the distribution of the cost of the minimum assignment when the matrix entries are independent identically distributed random variables.

In 1998 Giorgio Parisi [14] conjectured that for $\exp (1)$-distributed matrix entries, the expected minimum cost is

$$
1+\frac{1}{4}+\frac{1}{9}+\cdots+\frac{1}{n^{2}}
$$

This formula has subsequently been generalized in various directions $[3,4,8,7,10,13,12$, $15,16,17,20]$. The $\zeta(2)$ limit was proved by David Aldous [1] in 2000 and the formula (3.1) was established independently by Chandra Nair, Balaji Prabhakar, and Mayank Sharma [13] and by Svante Linusson and the author [10].

A key ingredient of the proof in [10] was the study of participation probabilities. We condition on a set $Z \subseteq[n] \times[n]$ of matrix entries being zero. The participation probability of a position $(i, j)$ is the probability that $(i, j)$ belongs to the minimum assignment. If there are several assignments of minimum cost, then the participation event may not be well-defined, but there are several circumstances under which it is well-defined with probability 1 , so that without ambiguity we can speak of the participation probability. For instance, this is the case if $(i, j) \notin Z$, or $(i, j)$ is the only element of $Z$ in its row or in its column.

The point of studying participation probabilities when trying to prove (3.1) was that if we know them for various sets $Z$, we can recursively compute the expected cost of the minimum assignment using Lemma 3.1 below. This lemma was proved in [11] and relies only on the special properties of the exponential distribution. In Section 7 we give a proof of the slightly more general Lemma 7.3. Given that the matrix has zeros in the positions in $Z$, let $Q(i, j, Z)$ denote the participation probability of $(i, j)$, and let $C(Z)$ denote the cost of the minimum assignment.

Lemma 3.1. If $(i, j) \notin Z$, then

$$
Q(i, j, Z)=\mathbb{E}(C(Z))-\mathbb{E}(C(Z \cup(i, j))) .
$$

Hence if we can compute the participation probabilities for all $Z$ and $(i, j)$, then we can recursively compute $\mathbb{E}(C(Z))$ by starting from sets $Z$ that contain an assignment and 


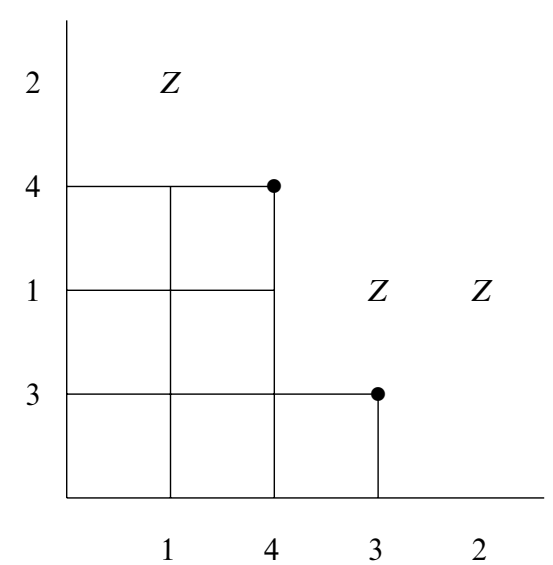

Figure 1. The urn process. The labels along the $x$-axis represent a uniform random permutation of the rows $1, \ldots, n$, while the labels along the $y$-axis represent a similar permutation of the columns. For $Z$ as in Example 2.1, the region $R(Z)$ and its outer corners $(4,4)$ and $(3,3)$ are indicated, as well as the points corresponding to the elements of $Z$.

working towards smaller $Z$. Ideally we can obtain (3.1) as

$$
\mathbb{E}(C(\emptyset))=Q\left(i_{1}, j_{1}, \emptyset\right)+Q\left(i_{2}, j_{2},\left\{\left(i_{1}, j_{1}\right)\right\}\right)+Q\left(i_{3}, j_{3},\left\{\left(i_{1}, j_{1}\right),\left(i_{2}, j_{2}\right)\right\}\right)+\cdots,
$$

where $\left(i_{1}, j_{1}\right),\left(i_{2}, j_{2}\right),\left(i_{3}, j_{3}\right), \ldots$ are the elements of $[n] \times[n]$ in any order.

In practice what one knows is that, for instance, the participation probabilities in a row or column must add to 1 , since always exactly one entry participates. This gives a linear relation among $\mathbb{E}(C(Z))$ for different $Z$, and the proof of (3.1) in [10] used certain combinatorial tricks to obtain further linear relations until $\mathbb{E}(C(\emptyset))$ could be solved for.

\section{The Buck-Chan-Robbins urn process}

The Buck-Chan-Robbins urn process was introduced in [3] and developed further in $[8,16,17,20]$. Originally it was used in the context of weighted and incomplete versions of the assignment problem, which we do not discuss here.

Proposition 4.1 below can in principle be deduced from the formulas of [10], although it is stated explicitly only in [16], in the considerably more general context of weighted incomplete flow problems.

In our context, the urn process simply consists of a random ordering of the rows and an independent random ordering of the columns, each chosen from the uniform distribution on the $n$ ! possible orderings. The orderings are displayed on the two axes of a coordinate system, as in Figure 1. We make the arbitrary choice of letting the rows occur on the $x$-axis and the columns on the $y$-axis.

The orderings of the rows and columns, together with $Z$, give rise to a random plane region that we call $R(Z)$. Notice first that each position $(i, j)$ in the matrix corresponds to a point whose coordinates have labels $i$ and $j$. The region $R(Z)$ is defined as the set of all points $(x, y)$ in the positive quadrant (and here we typically think of $x$ and $y$ as lying between the labels on the axes) such that we cannot find an assignment consisting only 
of positions $(i, j)$ such that $(i, j) \in Z$ or the label $i$ occurs to the left of $x$ or the label $j$ occurs below $y$. Figure 1 shows a possible outcome of the region $R(Z)$ with the choice of $Z=\{(1,2),(2,1),(3,1)\}$ as in Example 2.1.

For instance, in Figure 1, suppose $x$ is between the labels ' 4 ' and ' 3 ' on the $x$-axis, and $y$ is between ' 3 ' and ' 1 ' on the $y$-axis. We claim that $(x, y)$ is outside $R(Z)$. To verify this we must find an assignment, and we are allowed to use positions in $Z$, positions in rows 1 and 4 (because the labels 1 and 4 are to the left of $x$ ) and positions in column 3 (because the label 3 is below $y$ ). There are four possibilities. We assign rows 1 and 4 to columns 2 and 4 in one of the two ways, and then rows 2 and 3 to columns 1 and 3 in one of the two ways.

If on the other hand $x$ is between labels 1 and 4 on the $x$-axis, and $y$ is between labels 1 and 4 on the $y$-axis, we claim that $(x, y)$ is in $R(Z)$. In this case the only positions that are allowed are those in row 1 and in columns 1 and 3 , since this already includes everything in $Z$. An assignment of allowed positions is impossible since it would have to assign the three rows 2, 3 and 4 to only the two columns 1 and 3 .

The region $R(Z)$ has a staircase shape defined by its outer corners. Notice that if $Z$ is empty, then $R(Z)$ is a 'perfect' staircase shape with $n$ outer corners representing a uniform random bijection between the labels on the $x$-axis and the labels on the $y$-axis, in other words an assignment. If $Z$ is non-empty, then $R(Z)$ is a subset of $R(\emptyset)$, and may have fewer outer corners, although depending on the permutations of the labels it is still possible that $R(Z)=R(\emptyset)$ for some $Z$. In the example in Figure 1, the outer corners are the points whose coordinates have labels $(4,4)$ and $(3,3)$.

The following proposition is a special case of Lemma 9.3 of [16], and links the random assignment problem to the urn process.

Proposition 4.1. If $(i, j) \notin Z$, then the participation probability $Q(i, j, Z)$ is equal to the probability that there is an outer corner of $R(Z)$ labelled $(i, j)$.

At this point the reader may have guessed that we are going to link the assignment problem to the passenger model by a coupling of the passenger model and the urn process.

Proposition 4.2. If $(i, j) \notin Z$, then the probability $P(i, j, Z)$ that passenger $i$ receives seat $j$ in the passenger model is equal to the probability that there is an outer corner of $R(Z)$ labelled $(i, j)$ in the urn process.

Proof. In the passenger model, the behaviour of the passengers is unchanged if we assume that they follow a common random order of preferences on the seats (they of course still have to sit where they have a ticket if possible). The coupling is as follows. Given the outcome of the urn process, we let the passengers prefer seats in the order of the labels on the $y$-axis, so that the bottom label is the best seat and the top label is the worst. Moreover, we let the passengers enter in the reverse order of their labels on the $x$-axis, that is, from right to left.

We are going to show that under this coupling, the set of outer corners of $R(Z)$ is equal to the set $S$ of passenger-seat pairs without tickets resulting from the passenger model. 
First observe that if we identify $S$ with the corresponding points in the diagram, no point in $S$ is above and to the right of any other, because for any two passengers, if neither obtained a seat to which they had a ticket, then the passenger entering first must have taken a seat of higher preference than the passenger entering later.

Having established this, what remains is to show that $R(Z)$ is equal to the set of points lying below and to the left of some point in $S$. Therefore let $(x, y)$ be a point in the diagram. Again we assume that $x$ and $y$ are not exactly on the points of the labels. We want to show that $(x, y) \in R(Z)$ if and only if there is a passenger-seat pair $(i, j) \in S$ above and to the right of $(x, y)$, in other words the label $i$ on the $x$-axis is to the right of $x$, and the label $j$ on the $y$-axis is above $y$.

The point $(x, y)$ naturally defines a division of seats into good and bad, and of passengers into early and late. A seat is good if its label is below $y$ on the $y$-axis, and otherwise it is bad. Similarly a passenger whose label is to the right of $x$ is early, and if the label is to the left of $x$, he or she is late.

The statement that $(i, j)$ corresponds to a point above and to the right of $(x, y)$ is equivalent to $i$ being an early passenger and $j$ a bad seat. Notice that by definition, $(x, y) \in R(Z)$ if and only if every assignment of passengers to seats must put some early passenger in a bad seat to which they did not have a ticket.

Suppose first that $(x, y) \in R(Z)$. Then every assignment, and in particular the one resulting from the passenger model, must contain some passenger-seat pair $(i, j)$ such that $(i, j) \notin Z$, passenger $i$ is early, and seat $j$ is bad, which is what we wanted to prove.

Suppose on the other hand that $(x, y) \notin R(Z)$. This means that there is an assignment of passengers to seats such that no early passenger gets a bad seat to which they did not have a ticket. Since each passenger has at most one ticket, it is clear that in the passenger model, the early passengers will use all seats to which they collectively have tickets, and then as many as needed of the other seats in order of preference. Therefore no early passenger will choose a bad seat to which they do not have a ticket unless this is forced by the number of early passengers being larger than the number of seats that are either good or to which some early passenger has a ticket. But this cannot be the case, since by assumption there is some assignment that avoids early passengers getting bad seats to which they did not have tickets. It follows that the assignment resulting from the passenger model must be such an assignment, and this establishes the proposition.

In the example from Figure 1, the passengers like seat 3 best, then seats 1, 4, and 2 in this order. They enter the aircraft in the order 2, 3, 4, 1. Passenger 2, who enters first, has a ticket to seat 1 and therefore picks this seat. Then passenger 3, who also has a ticket to seat 1 , enters. Since seat 1 is occupied, passenger 3 picks seat 3 , which is the best seat according to the common order of preference. Then passenger 4 enters and picks seat 4 , which is the best seat which is still free. Finally passenger 1 enters, and by coincidence gets the seat to which she had a ticket. The resulting passenger-seat pairs not corresponding to tickets are $(3,3)$ and $(4,4)$, and these are also the outer corners of $R(Z)$.

Corollary 4.3. If $(i, j) \notin Z$, then $P(i, j, Z)=Q(i, j, Z)$. 
Actually the proof of Proposition 4.2 shows more: not only is the probability of a certain passenger-seat pair $(i, j) \notin Z$ being an outer corner of $R(Z)$ the same as the probability of passenger $i$ being assigned seat $j$ in the passenger model, but the set of outer corners in $R(Z)$ has the same distribution as the set of unticketed passenger-seat pairs resulting from the passenger model. This hints at the following more general conjecture.

Conjecture 4.4. The set of non-zero positions in the minimum assignment has the same distribution as the set of outer corners in the urn process.

This conjecture can in turn be generalized to weighted flow problems [20], and polymatroid problems [8], which we do not discuss here. It is part of the 'programme' of understanding the connection between optimization problems and urn models. If Conjecture 4.4 could be established and generalized in the proper way to 2-factor problems as in [6], then possibly one could establish that the number of cycles in the minimum 2 -factor is $O(\log n)$. As is explained in [6], this would greatly simplify the analysis of the random travelling salesman problem in $[6,20]$.

We do not have much substantial evidence for Conjecture 4.4 apart from the fact that it is consistent with the participation probabilities of individual matrix entries. It is also consistent with simulations for $n=3$ but has not been tested further. On the other hand it would be very strange if the two probability distributions turned out to be different and only 'by coincidence' assign the same total probability to individual matrix entries.

\section{Proof of the Parisi formula}

We show that the Parisi formula (3.1) follows from Corollary 4.3. However, this does not give a new proof of (3.1), since Corollary 4.3 relies on the results of [16].

Theorem 5.1. The expected cost of the minimum assignment in an $n$ by $n$ matrix with independent $\exp (1)$ entries is $1+1 / 4+\cdots+1 / n^{2}$.

Proof. Let $k$ be an integer such that $1 \leqslant k \leqslant n$. We let $Z=\{(1,1), \ldots,(k-1, k-1)\}$, and compute the probability $P(k, k, Z)$. When passenger $k$ enters the aircraft, the number of passengers among the first $k-1$ that have already boarded is uniformly distributed on $0, \ldots, k-1$. Suppose this number is $r$. Then the seats of those $r$ passengers must be occupied (whether or not by the passenger with a ticket to that seat). Since the passengers without tickets, or whose seats were occupied, have been seated uniformly on the remaining seats, the probability that passenger $k$ sits down in seat $k$ is by symmetry $1 /(n-r)$. Therefore

$$
P(k, k, Z)=\mathbb{E}\left(\frac{1}{n-r}\right)=\frac{1}{k}\left(\frac{1}{n}+\frac{1}{n-1}+\cdots+\frac{1}{n-k+1}\right),
$$

where we take expectation over $r$ uniformly distributed on $\{0, \ldots, k-1\}$ in the middle expression. 
By Lemma 3.1, the expected cost of the minimum assignment is obtained by summing these numbers for $k=1, \ldots, n$. We then obtain the following formula, which is a special case of the Coppersmith-Sorkin formula $[4,10,13]$ :

$$
\sum_{\substack{1 \leqslant p, q \leqslant n \\ p+q>n}} \frac{1}{p \cdot q}
$$

It is easily proved by induction on $n$ that (5.1) equals the Parisi formula (3.1): when $n$ increases to $n+1$, the terms $1 / p q$ for which $p+q=n+1$ are replaced by $1 / p(n+1)+$ $1 / q(n+1)$, so the net increase is the term $1 /(n+1)^{2}$.

We also obtain a formula for the expected cost of the minimum assignment given that there is a partial assignment of zero cost. If there are $k$ zeros in different rows and columns, then this expected cost is

$$
\mathbb{E}(C(\{(1,1), \ldots,(k, k)\}))=\sum_{\substack{1 \leqslant p \leqslant n \\ k<q \leqslant n \\ p+q>n}} \frac{1}{p \cdot q} .
$$

It is remarkable that this is the same as the expected cost of the minimum path tree to $n-k$ vertices in the complete graph on $n+1$ vertices, as follows by putting $N=n+1$ and $m=n-k$ in equation (11) of [9]. For $k=n-1$ there is a simple explanation (see [18]), but for smaller $k$, there seems to be no obvious reason for this fact. For $k=0$, it is known that the two quantities do not have the same variance [9, 17]. However, it is shown in [18] that conditioning on an edge belonging to the shortest path tree, its cost is distributed in the same way as the cost of a matrix entry in the assignment problem given that it belongs to the minimum assignment. Finding the correct generalization remains an interesting problem.

\section{Generalization of the passenger model}

The urn model has an obvious symmetry under reversing the roles of rows and columns of the matrix. In the passenger model, the assumption that no passenger has more than one ticket destroys this symmetry.

It is possible to obtain a symmetric version of the passenger model allowing any set $Z$ of tickets, and even to generalize it further, but at some point the generalizations become meaningless as they only boil down to the urn model as described in $[16,20]$ without simplifying the reasoning about it. We briefly describe how to allow passengers to have tickets to several seats, but this is already a bit artificial. It is based on interpreting the outer corners of $R(Z)$ as passengers choosing seats.

The rule is that passengers who sit in seats to which they have tickets are willing to move to other seats to which they have tickets, if other passengers arriving later want their seats. Even several passengers sitting in seats to which they have tickets are willing to simultaneously change to other seats to which they have tickets, in order to free a seat which is preferred by a passenger boarding later. One way of thinking about this 
is that passengers who can choose between several seats to which they have tickets will not decide immediately in which of them to sit. They will instead restrict the following passengers' choices only by claiming to sit in one of their seats.

It is also obvious how to generalize the passenger model to seating $m$ passengers in $n$ seats, $m \leqslant n$, but this again destroys the symmetry. We refrain from describing the again symmetric model for seating $k$ out of those passengers.

\section{An extremal result}

We can use the generalized passenger model to prove the following result of extremal nature.

Theorem 7.1. Let $Z \subseteq[n] \times[n]$, and suppose that $(1,1) \notin Z$. Then

$$
P(1,1, Z) \leqslant \frac{1+1 / 2+\cdots+1 / n}{n} .
$$

Proof. When passenger 1 enters the aircraft, the number $k$ of free seats is uniformly distributed on $1, \ldots, n$. Either she can take a seat to which she has a ticket, or else all the free seats (and possibly some of the occupied seats) are available to her. In either case, the probability that she chooses seat 1 is at most $1 / k$.

Notice that if $Z=\{(2,2), \ldots,(n, n)\}$, then the value on the right-hand side of $(7.1)$ is attained.

It was shown in the preprint [17] that the variance of the cost of the minimum assignment is asymptotically $c / n$ as $n \rightarrow \infty$, where $c=4 \zeta(2)-4 \zeta(3)$. The starting point that eventually led to [17] was an idea of using results like Theorem 7.1 in order to bound the variance of the cost of random optimization problems.

The approach has so far not succeeded, but is potentially applicable to a wider range of problems, and is worth describing briefly. Let us consider a slightly more general setting than the assignment problem. Let $x_{1}, \ldots, x_{N}$ be independent random variables of mean 1 exponential distribution. Let $A$ be a family of subsets of $\{1, \ldots, N\}$. Then we consider the optimization problem

$$
C=\min _{S \in A} \sum_{i \in S} x_{i} .
$$

The assignment problem corresponds to taking $N=n^{2}$ and letting $A$ be the family of the $n$ ! assignments.

Without any substantial evidence, we propose the following conjecture.

Conjecture 7.2. Except in trivial cases where $C=0$, there exists an $i \in\{1, \ldots, N\}$ such that the event that $x_{i}$ participates in the minimum solution is non-positively correlated with its $\operatorname{cost} C$. 
In analogy with the notation we used earlier, for $Z \subseteq\{1, \ldots, N\}$ let

$$
C(Z)=\min _{S \in A} \sum_{i \in S \backslash Z} x_{i}
$$

in other words the minimum conditioning on $x_{i}=0$ for all $i \in Z$. We call this a subproblem of (7.2).

The following generalization of Lemma 3.1 (which is used in $[17,19,20]$ ) also holds in this setting, and for completeness we provide a proof.

Lemma 7.3. Let $Z \subseteq\{1, \ldots, N\}$. Suppose that $i \notin Z$ and let $Z^{\prime}=Z \cup\{i\}$. Moreover, let $I$ be the indicator variable for the event that $x_{i}$ participates in the minimum solution to the subproblem given by $Z$. Then

$$
\mathbb{E}(I)=\mathbb{E}(C(Z))-\mathbb{E}\left(C\left(Z^{\prime}\right)\right),
$$

and

$$
2 \mathbb{E}(C(Z) \cdot I)=\mathbb{E}\left(C(Z)^{2}\right)-\mathbb{E}\left(C\left(Z^{\prime}\right)^{2}\right) .
$$

Proof. We condition on the values of all variables except $x_{i}$. Let $f(x)$ be the value that $C(Z)$ takes for $x_{i}=x$. We can express the quantities occurring in (7.4) and (7.5) in terms of $f$ and the random variable $x_{i}$. Since we are conditioning on all variables except $x_{i}$, $C\left(Z^{\prime}\right)$ is now the non-random quantity $f(0)$. Moreover, $C(Z)=f\left(x_{i}\right)$ and $I=f^{\prime}\left(x_{i}\right)$, since $f$ is a piecewise linear function with slope 1 in the range where $x_{i}$ participates in the minimum, and slope 0 in the range where $x_{i}$ is too large to participate.

What equations (7.4) and (7.5) are saying is therefore that

$$
\mathbb{E} f^{\prime}\left(x_{i}\right)=\mathbb{E} f\left(x_{i}\right)-f(0)
$$

and

$$
\mathbb{E}\left(2 f^{\prime}\left(x_{i}\right) f\left(x_{i}\right)\right)=\mathbb{E}\left(f\left(x_{i}\right)^{2}\right)-f(0)^{2} .
$$

If $g$ is a continuous and piecewise differentiable function such that

$$
\lim _{x \rightarrow \infty} e^{-x} g(x)=0
$$

then by partial integration,

$$
\mathbb{E} g^{\prime}\left(x_{i}\right)=\int_{0}^{\infty} e^{-x} g^{\prime}(x) d x=\int_{0}^{\infty} e^{-x} g(x) d x-g(0)=\mathbb{E} g\left(x_{i}\right)-g(0) .
$$

Here (7.6) follows by taking $g=f$, and (7.7) by taking $g=f^{2}$.

Now let $\sigma$ be the maximum participation probability of any variable in any subproblem of (7.2), which by Lemma 7.3 is

$$
\sigma=\max _{Z \subseteq\{1, \ldots, N\}} \max _{i \notin Z} \mathbb{E} C(Z)-\mathbb{E} C(Z \cup\{i\}) .
$$

For the assignment problem, $\sigma$ is given by the right-hand side of (7.1). 
Proposition 7.4. If Conjecture 7.2 holds for every subproblem of (7.2), then

$$
\operatorname{var}(C) \leqslant \sigma \cdot \mathbb{E} C
$$

Proof. Let $Z \subseteq\{1, \ldots, N\}$ and let $Z^{\prime}=Z \cup\{i\}$. Moreover, let $I$ be the indicator variable for the event that $x_{i}$ participates in the minimum solution to the subproblem given by $Z$. According to Conjecture 7.2, if $i$ is chosen correctly depending on $Z$, then

$$
\mathbb{E}(C(Z) \cdot I) \leqslant \mathbb{E}(C(Z)) \cdot \mathbb{E}(I) .
$$

Using equations (7.4) and (7.5) to replace all expectations of squares by variances, we obtain

$$
\operatorname{var}(C(Z)) \leqslant \operatorname{var}\left(C\left(Z^{\prime}\right)\right)+\left[\mathbb{E}(C(Z))-\mathbb{E}\left(C\left(Z^{\prime}\right)\right)\right]^{2} .
$$

Since $\mathbb{E}(C(Z))-\mathbb{E}\left(C\left(Z^{\prime}\right)\right) \leqslant \sigma$, we have

$$
\operatorname{var}(C(Z)) \leqslant \operatorname{var}\left(C\left(Z^{\prime}\right)\right)+\sigma \cdot\left[\mathbb{E}(C(Z))-\mathbb{E}\left(C\left(Z^{\prime}\right)\right)\right] .
$$

By induction we may assume that $\operatorname{var}\left(C\left(Z^{\prime}\right)\right)-\sigma \cdot \mathbb{E}\left(C\left(Z^{\prime}\right)\right) \leqslant 0$, by which it follows from (7.9) that $\operatorname{var}(C(Z)) \leqslant \sigma \cdot \mathbb{E}(C(Z))$ as claimed.

Thus, if Conjecture 7.2 had been established, it would have given a bound of $O(\log n / n)$ for the variance of the cost of the assignment problem, but this was superseded by the $O(1 / n)$ bound in [17]. Conjecture 7.2 is still of interest, as there is hope of establishing analogues of Theorem 7.1 for problems where the method of [17] does not apply.

If there is a non-empty set $U \subseteq\{1, \ldots, N\}$ of which a constant (non-random) number $k$ of variables must participate in the minimum in (7.2), then it is easy to see that Conjecture 7.2 will hold for some $i \in U$ : if $I_{i}$ is the indicator variable for the event that $x_{i}$ participates in the minimum, then

$$
\sum_{i \in U} \mathbb{E}\left(C \cdot I_{i}\right)=\sum_{i \in U} \mathbb{E}(C) \cdot \mathbb{E}\left(I_{i}\right)
$$

both sides being equal to $k \cdot \mathbb{E}(C)$. Clearly not every term in the first sum can be larger than the corresponding term in the second sum.

For instance, if in the assignment problem there is a row or column that does not intersect $Z$, then exactly one of its entries is in the minimum assignment, and Conjecture 7.2 must hold. The same holds for instance if $n=3$ and $Z=\{(1,2),(1,3),(2,1),(3,1)\}$. Then the minimum assignment must contain exactly one of the entries $(2,2),(2,3),(3,2)$ and $(3,3)$. It is easy to verify in this way that Conjecture 7.2 holds for $n \leqslant 4$ and all $Z$. However, if $n$ is large enough there will be a $Z$ for which no $U$ has this property.

Incidentally, the existence of such sets $U$ also allows a recursive computation of $\mathbb{E}(C(Z))$ using Lemma 3.1. The fact that such a set does not always exist was therefore also the reason why the method employed in $[2,4,11]$ did not lead to a proof of $(3.1)$. 


\section{The generalized Dotsenko conjecture}

In [5], Viktor Dotsenko presented what was at the time a quite interesting conjecture, indeed the first purely qualitative conjecture that implied the Parisi formula (all previous conjectures were formulas motivated only by extensive verification of examples). Unfortunately Dotsenko incorrectly claimed to have proved the conjecture, and the paper was therefore largely ignored.

Dotsenko does not seem to have been aware of Lemma 3.1, but used ideas similar to those of $[4,11]$. He considered differences in expected value of assignment problems with various configurations of zero entries, and his main conjecture can be reinterpreted in terms of participation probabilities. We consider a matrix with $m$ rows and $n$ columns $(m \leqslant n)$, where the objective is to minimize the sum of a set of $m$ matrix entries under the constraint of one entry in each row and no two in the same column.

Proposition 8.1 (Dotsenko's conjecture). Let $k<n$, and let

$$
Z=\{(1,1), \ldots,(k, k)\} .
$$

Then the participation probabilities in rows $k+1, \ldots, m$ depend on $k$ and $n$ but not on $m$.

In other words, if we add another row (without zeros) to the matrix, then the participation probabilities in the rows without zeros will still be the same. Dotsenko correctly proved that this would imply the Parisi formula (3.1), with an argument similar to the one in Section 5. The mistake in [5] occurs in Appendix A, where he claims to establish Proposition 8.1.

The paper was frowned upon by mathematicians, in particular since Dotsenko is a physicist, but the statement of Proposition 8.1 is so simple that there is reason to believe that, besides [19], another short proof of the Parisi conjecture (3.1) has been overlooked. We prove that Dotsenko was right, by establishing the following generalization, allowing several zeros in the same column.

Theorem 8.2 (generalized Dotsenko conjecture). If $Z$ contains at most one position in each row, then the participation probabilities in the rows without zeros depend on $k$ and $n$ but not on $m$.

This also follows from the results established by C. Nair in Section 3.4 of [12].

Proof. Consider the passenger model with $m$ passengers and $n$ seats, $m \leqslant n$. Suppose that $Z$ contains one zero in each of the first $k$ rows, equivalently, passengers $1, \ldots, k$ have tickets (possibly to the same seats), and the remaining passengers have no tickets. Consider a passenger $i$ without a ticket. Let $r$ be the number of passengers of the first $k$ who entered before passenger $i$. Regardless of $m, r$ is uniformly distributed on $0, \ldots, k$. Now condition on the set $S$ of passengers among $1, \ldots, k$ who entered before passenger $i$. The set $S$ has the same distribution regardless of $m$. The seats to which the passengers in $S$ collectively have tickets will be occupied by the time passenger $i$ enters (whether 
or not by passengers who have tickets to those seats). By symmetry, the distribution of occupied seats among the remaining seats, regardless of the number of passengers who have entered, will be uniform on these seats. Therefore passenger $i$ will end up in a seat uniformly distributed among the seats to which nobody in $S$ has a ticket. In view of Corollary 4.3 , this proves the claim.

It is easy to show by example that the assumption that $Z$ contains at most one position in each row is necessary. Therefore Theorem 8.2 seems to be the correct generalization. A simple proof would be very interesting.

\section{Buying the tickets}

In view of the proof of the Parisi conjecture, Theorem 5.1, it is natural to think of $P(i, j, Z)$ as the price of a ticket to seat $j$ for passenger $i$. Imagine a seller of $n^{2}$ personal tickets, one for each passenger-seat pair. The passengers, who somehow prefer to have a ticket to the seat where they end up, buy the tickets one by one, and the price of ticket $(i, j)$, which changes according to the 'market' is given by $P(i, j, Z)$, where $Z$ is the set of tickets that are already bought.

Although far from realistic, this model is reasonable in some respects. When the first ticket has been sold, other tickets for the same passenger or the same seat lose in value due to decreasing 'demand', and as soon as $Z$ contains a complete assignment of passengers to seats, remaining tickets become worthless.

The following is a consequence of the connection to the minimum assignment problem.

Proposition 9.1. The price of buying all tickets is independent of the order in which they are bought.

Proof. It follows from Lemma 3.1 and Corollary 4.3 that the sum of the ticket prices is a telescoping sum of $\mathbb{E}(C(Z))$, so that for any $Z$, the price of buying all remaining tickets is $\mathbb{E}(C(Z))$.

It would be interesting to have a simple explanation of Proposition 9.1 without reference to the minimum assignment problem.

\section{Acknowledgement}

I thank an anonymous referee for valuable comments on a earlier version of the paper.

\section{References}

[1] Aldous, D. (2001) The $\zeta(2)$ limit in the random assignment problem. Random Struct. Alg. 18 381-418.

[2] Alm, S. E. and Sorkin, G. B. (2002) Exact expectations and distributions for the random assignment problem. Combin. Probab. Comput. 11 217-248. 
[3] Buck, M. W., Chan, C. S. and Robbins, D. P. (2002) On the expected value of the minimum assignment. Random Struct. Alg. 21 33-58.

[4] Coppersmith, D. and Sorkin, G. B. (1999) Constructive bounds and exact expectations for the random assignment problem. Random Struct. Alg. 15 133-144.

[5] Dotsenko, V. S. (2000) Exact solution of the random bipartite matching model. J. Phys. A 33 (10), 2015-2030.

[6] Frieze, A. M. (2004) On random symmetric travelling salesman problems. Math. Oper. Res. 29 878-890.

[7] Hessler, M. and Wästlund, J. (2009) LP-relaxed matching with free edges and loops. In 'Optimization, matroids and error-correcting codes', PhD thesis of M. Hessler, Linköping, Sweden.

[8] Hessler, M. and Wästlund, J. (2010) Edge cover and polymatroid flow problems. Electron. J. Probab. 15, 2200-2219.

[9] van der Hofstad, R., Hooghiemstra, G., and Van Mieghem, P. (2006) Size and weight of shortest path trees with exponential link weights. Combin. Probab. Comput. 15 903-926.

[10] Linusson, S. and Wästlund, J. (2004) A proof of Parisi's conjecture on the random assignment problem. Probab. Theory Rel. Fields 128 419-440.

[11] Linusson, S. and Wästlund, J. A generalization of the random assignment problem. arXiv: math.CO/0006146.

[12] Nair, C. (2005) Proofs of the Parisi and Coppersmith-Sorkin conjectures in the random assignment problem. PhD thesis, Stanford.

[13] Nair, C., Prabhakar, B. and Sharma, M. (2005) Proofs of the Parisi and Coppersmith-Sorkin random assignment conjectures. Random Struct. Alg. 27 413-444.

[14] Parisi, G. (1998) A conjecture on random bipartite matching. arXiv: cond-mat/9801176.

[15] Wästlund, J. (2005) A proof of a conjecture of Buck, Chan and Robbins on the expected value of the minimum assignment. Random Struct. Alg. 26 237-251.

[16] Wästlund, J. (2005) Exact formulas and limits for a class of random optimization problems. Linköping Studies in Mathematics no. 5.

[17] Wästlund, J. (2005) The variance and higher moments in the random assignment problem. Linköping Studies in Mathematics no. 8.

[18] Wästlund, J. (2006) Random assignment and shortest path problems. In Proc. Fourth Colloquium on Mathematics and Computer Science, September 2006, Institut Élie Cartan, Nancy, France.

[19] Wästlund, J. (2009) An easy proof of the zeta(2) limit in the assignment problem. Electron. Comm. Probab. 14 261-269.

[20] Wästlund, J. (2010) The mean field traveling salesman and related problems. Acta Math. 204 91-150. 